Article

\title{
Diversification of Municipalities Located in the Impact Area of National Parks in Terms of Environmental Requirements of Sustainable Tourism
}

\author{
Agata Pawłat-Zawrzykraj * (1) and Konrad Podawca \\ Institute of Environmental Engineering, Faculty of Civil and Environmental Engineering, Warsaw University of \\ Life Science-WULS, 02-787 Warsaw, Poland; konrad_podawca@sggw.edu.pl \\ * Correspondence: agata_pawlat_zawrzykraj@sggw.edu.pl
}

Received: 13 May 2020; Accepted: 12 June 2020; Published: 16 June 2020

check for updates

\begin{abstract}
The main objective of the paper was to show the diversification of Polish municipalities that have national parks within their boundaries in terms of implementing sustainable tourism priorities. The study focused on ecological and environmental aspects, primarily related to the shaping and maintenance of green areas, as well as waste and wastewater management. The assessment was based on statistical data taken from the Local Data Bank for the years 2012-2018. The authors determined their own set of indicators, describing green areas management, the environmental risk associated with waste and wastewater generation, and the reshaping of the forest and agricultural landscape. The obtained results were compared with the spatial diversification of the surveyed administrative units in terms of tourist attractiveness carried out by us in 2018. The study made it possible to indicate, among others, municipalities that are prime tourist destinations and have highly developed tourist facilities, but do not keep up with sustainable tourism activities. There are also units that carry out activities in the field of forest and agricultural land protection, invest in public green areas, properly manage sewage and wastewater, and, at the same time, are not attractive for tourists.
\end{abstract}

Keywords: national park; sustainable tourism; environmental protection; sustainable tourism indicators; local spatial policy

\section{Introduction}

The number of tourists visiting national parks (NP) in Poland is constantly growing. In 2001, 10.345 million visitors were reported; in 2005, 10.525 million; in 2014, 11.799 million; and in 2018, 14.035 million [1]. We have 23 national parks and the spatial distribution of objects with the largest number of tourists is uneven. Three of the six parks visited by a million or more tourists in 2018 are located in mountainous regions (Tatra NP-3.97 million; Karkonosze NP_-2.0 million; and Góry Stołowe NP-1.06 million), one is in the coastal region (Wolin NP-1.5 million), and two of them are located in zones directly affected by large cities and "combine the touristic and suburban recreation functions" [2], namely, Wielkopolska NP and Kampinos NP (1 million each).

The impact of the existence of national parks on the condition of the natural environment in protected areas and increasing environmental awareness of their users is highly rated $[3,4]$. There is no doubt that administrative units located within the boundaries of national parks attract tourists, both in Poland and abroad. This is confirmed in the literature concerning factors that determine tourists' choices of holiday destinations [4-9] and research on tourism potential and attractiveness [2,5-7].

A large and constantly growing number of tourists generates significant income for the park itself, as well as for the administrative units where national parks are located and for the residents and providers of tourist services [8]. At the same time, tourism is a source of threats and nuisances to the 
nature of a park and its neighbouring areas. In the case of some national parks, we can already state that we are dealing with the phenomenon of "overtourism" - a situation where tourism development overwhelms local ecological, physical, social, and economic capacity $[4,5,9]$. Therefore, a significant number of authors state that tourism in national parks stands in conflict with the environmental protection objectives defined for these areas [10-12]. One of the basic measures for the assessment of such a threat is tourist capacity, beyond which there is a risk of temporary or irreversible degradation of the natural resources. Another criterion of the environmental costs of tourism may also be the amount of additional waste and sewage, as well as traffic pollution generated by tourists.

Tourism in protected areas is specific seeing as how it should be implemented in a sustainable manner. According to the definition of the Federation of National Parks and Nature Reserves, this translates to "any form of tourism development, management and tourist activity that supports ecological, social and economic integrity of areas, while preserving the natural and cultural resources of these areas" [13]. It is a form of gentle or environmentally friendly tourism. It means activity, or rather management, which tries to balance the needs and expectations of tourists, the local community, and the needs of the natural environment $[14,15]$. These general priorities for sustainable tourism can be further specified by the following principles:

- adjustment of tourism to the resources, quality, and capacity of the environment;

- utilization of local natural, human, and material resources in tourism services;

- adaptation of tourist facilities to the character of the area, fitting in with the local natural and cultural landscape;

- integration of tourism with local spatial and social development so that it is socially accepted and beneficial for the local community [16].

The objectives formulated this way are difficult to achieve and require seeking a compromise between various interest groups. Quoting a guidebook of the World Tourism Organization (WTO): "Tourism sector decision-makers need to know the links between tourism and the natural and cultural environments, including the effects of environmental factors on tourism (possibly expressed as risks to tourism) and the impacts of tourism on the environment" [17]. This is especially the case in areas under nature protection.

The issue of sustainable tourism in national parks and areas administratively related to this form of nature protection is rather complex. It is a form of activity that not only provides benefits but is also a source of threats and various environmentally negative impacts [12]. This is why it is and should continue to be an object of common interest to park authorities, local governments, and residents. There is a need to build on the knowledge regarding the relationship between tourism development and the natural, economic, and social conditions of the spatial management of the national parks and areas administratively and functionally connected to them $[18,19]$.

Decision-making in sustainable tourism planning and management can be supported by various tools. The WTO has been promoting the use of sustainable tourism indicators (STIs) since the early 1990s, as essential instruments for policy-making, planning, and management processes at the destinations [17]. STIs were generically assigned to economic, planning, social, and ecological indicator types. The literature [20-22] presents a similar division into the following core groups:

1. destination management indicators-described, e.g., by customer satisfaction;

2. economic indicators-described, e.g., by the volume and value of the tourism flow at the destination, performance of the tourism enterprise(s), quantity and quality of tourism employment, and the tourism supply chain;

3. local socio-cultural impacts—indicators described, e.g., by the percentage of residents who are satisfied with the tourism at the destination, the percentage of residents that are satisfied with the impacts of tourism on the destination's identity, and the percentage of the events in the destination that are focused on traditional/local culture and heritage, protecting and enhancing the cultural heritage, local identity, and assets; 
4. environmental impact indicators-describing the impact of transport, air quality, solid waste management, sewage treatment, water consumption, energy usage, and activities focused on landscape and biodiversity protection.

It is interesting to note that, according to Diamond [23], regional councils showed a preference towards ecological indicators. Territorial local authorities and regional tourism organizations preferred economic and social indicators.

The use of STIs raises several issues, mainly because of the multiple interpretations of the concept of sustainable development, and by extension of the concept of sustainable tourism. Some of the methods or evaluation criteria are being questioned. Among other things, this is the result of the discrepancy between the approach applied by the academic community and the needs and goals expressed by authorities at different decision-making levels $[24,25]$. The selection of indicators also depends on the destination because "many indicators cannot be calculated for a specific tourist region-and even less so for the local scale. In some cases, calculation concerns areas that are not necessarily compatible with the administrative territorial division of the tourist destination region" [25]. This is particularly the case in areas of natural protection and became an important issue in the present study.

Sustainable tourism indicators have been implemented in several studies concerning national parks. Huhtala et al. [26,27] focused on the local economic impacts of Finnish national parks, especially on local income and employment effects. Reihanian et al. [28] adapted the indicators for monitoring sustainability of tourism development in Boujagh National Park. The study focused on visitor satisfaction, the economy, environment, and society. Mihanyar et al. [29] studied the effects of national park sustainability on national park behavioural intention.

Research concerning sustainable tourism indicators also has been conducted in Poland. Kowalczyk [20], in an overview of the many indicators for monitoring sustainable development and sustainable tourism, pointed out the lack of a single, universally accepted method. Myga-Piatek and Jankowski [30] studied the topic of tourism and sustainable development in mountain national parks. The positive and negative influence of tourism on the environment and the landscape was presented in relation to legal conditions, regional strategies of development, and the state pro-ecological policy. Kruczek and Przybyło-Kisielewska [30] calculated indicators of tourist traffic density in the most crowded national parks in Poland. The main purpose of monitoring tourism was to take actions aimed at limiting the negative effects of excessive attendance. A similar study was performed by Rogowski [31] for Góry Stołowe National Park. Mazurkiewicz [32] developed a simple evaluation method assuming that sustainable tourism takes place when the sum of the related advantages is larger than that of the disadvantages from the viewpoint of various sets of interest groups. Zawilińska and Mika [3] assessed the role and impact of Polish national parks on local socio-economic development. They pointed out that "it was necessary to see a national park as a system linked to the socio-economic environment and to take planning actions based on a holistic look at natural, social and economic issues of a national park and its neighbourhood." This is an approach that requires, among others, increased social participation in shaping the development of these areas and strengthened cooperation between local authorities, the parks' management, non-governmental organizations, and local tourism businesses.

\section{Objectives, Study Area, Materials, and Methods}

The primary objective of the work was to show the diversification of municipalities located in the impact area of national parks in terms of implementing sustainable tourism priorities. "The impact area" is hereby understood as the area designated by the boundaries of administrative units in which the national park is located. This is an area where the spatial policy is shaped not only by local (municipal) development determinants, but also strongly affected by the priorities/objectives pursued by the national park.

The specific objectives were to examine the administrative units in terms of ecological and environmental aspects of sustainable tourism, especially related to 
- the shaping and maintenance of green areas;

- waste management;

- wastewater management.

This preliminary study covered 114 (4.5\%) of the 2477 municipalities in Poland. These are the administrative units that have been granted natural protection as national parks. In terms of area, the largest group ( $43 \%$ of units) are municipalities where the share of the national park area in the total unit area is less than $10 \%$. A quantitatively comparable group of units (37\%) comprises municipalities with a share of 10-30\%. Municipalities with an NP area share of 30-50\%, as well as municipalities with a share exceeding $50 \%$, each constitute groups that represent $10 \%$ of all studied units [33]. Out of all the municipalities that have national parks within their boundaries, 11 urban units have been eliminated, concluding that the development of tourism in these areas is driven by some additional factors and the obtained indicators may disturb the accurate picture of other units. The spatial scope of the study covered 103 rural and urban-rural municipalities.

In this study, the following research methods were applied:

- filtering data collected in the Local Data Bank (BDL) [34] in the fields of: tourism, state, and environmental protection and population;

- determination of indicators for the selected features to achieve their comparability;

- normalization of indicators in order to determine their significance in the implementation of sustainable tourism;

- designation of a synthetic indicator describing the rate of sustainable tourism implementation in terms of sewage and waste management, and green area management.

The results of the analysis were confronted with the spatial diversification of the surveyed administrative units in terms of tourist attractiveness carried out by us in 2018 [7]. In the quoted assessment, the so-called "tourist attractiveness" of the municipalities reflects the touristic use of the area. It was calculated on the basis of data such as the number of tourists, the number of tourist accommodation sites and beds, the number of overnight stays, and the utilization of the existing tourist infrastructure.

The selection of parameters that are meaningful for assessing the level of sustainable tourism in the studied municipalities was preceded by an analysis of the set of indicators proposed in the literature. The study focused on environmental issues, primarily related to sustainable tourism objectives such as

- compliance of tourism with natural conditions, among others through activities aimed at the protection of the landscape and green areas and preservation of the natural environment as a whole;

- development of tourism activity in an integrated way with proper spatial planning;

- $\quad$ limiting the amounts of waste and sewage [21,35-38].

To assess the implementation level of the selected aspects of sustainable tourism, statistical data taken from the BDL for the years 2012-2018 were used. In case of waste and wastewater management, priority was given to data describing quantities of waste and wastewater. It was assumed that tourists contribute to their production. In addition, the share of septic tanks supplying residential and housing/service buildings was taken into account as a source of risk to the quality of the soil and water resources. Therefore, the risk of environmental pollution associated with the generation of waste and wastewater was presented by the following indicators:

- the amount of municipal sewage in relation to the number of inhabitants and tourists $\left(\right.$ dam $^{3}$ per capita) $-\mathrm{X}_{1}$;

- the amount of waste generated during the year in relation to the number of inhabitants and tourists (t per capita) $-\mathrm{X}_{2}$; 
- the number of septic tanks in relation to the number of residential buildings and buildings with housing-service functions (-)- $\mathrm{X}_{3}$.

The values of indicators $X_{1}$ and $X_{2}$ calculated for each year were averaged, yielding mean values for the years 2012-2018. In case of the $X_{3}$ indictor, 2018 data were used. Additionally, a conversion number of tourists was used, which is the quotient of the number of overnight stays over 365 days. The value corresponds to the number of tourists who would stay in a given municipality throughout the year.

The risk associated with the reshaping of the forest and agricultural landscape, especially with changes in their designated use to new residential, industrial, service, and transport functions, was described by the following indictors:

- the total area of agricultural land (ha) in a municipality subject to transformation for non-agricultural purposes in the local spatial development plans, compared to the total area of agricultural land in the municipality (ha) $-\mathrm{X}_{4}(-)$;

- the total area of forest land (ha) in a municipality subject to transformation for non-forest purposes in the local spatial development plans, compared to the total area of forest land in the municipality (ha) - $\mathrm{X}_{5}(-)$.

The reduction of forest and agricultural areas can be partly compensated, amongst others, by increasing investments in establishing new green areas or the preservation and enlargement of the existing ones. In order to indicate these kinds of activities in the analysed municipalities, the following indicators were proposed:

- expenditures on the maintenance of green areas related to the total area of public green areas- $\mathrm{X}_{6}$ (PLN/ha);

- public green areas (parks, greenery, and housing estate greenery) related to the number of inhabitants and tourists $-X_{7}$ (ha per capita).

The next stage of the work was the normalization of the indicators, which made it possible to transform their values expressed in different units into a comparable form. Indexes $X_{6}$ and $X_{7}$, which have a stimulant character, were normalized in accordance with the following Equation (1):

$$
z_{i j}=\frac{x_{i j}}{\operatorname{maxx}_{i j}}
$$

Other indicators that have a destimulant character were normalized according to Equation (2):

$$
z_{i j}=1-\frac{x_{i j}}{\max x_{i j}}
$$

where:

$x_{i j}$ - the value of $j_{\text {th }}$ indicator in the $\mathrm{i}_{\text {th }}$ municipality;

maxx $_{i j}$ - the maximum value of $j_{\text {th }}$ indicator;

$z_{i j}$-the normalized value of $x_{i j}$.

Such an approach allowed a synthetic picture of the municipalities to be obtained in two subject areas, i.e., in the environmental and infrastructural aspects $\left(Z_{1 i}, Z_{2 i}, Z_{3 i}\right)$, as well as in the environmental and planning aspects $\left(Z_{4 i}, Z_{5 i}, Z_{6 i}, Z_{7 i}\right)$. The synthetic indicator of the environmental-infrastructural threats and environmental-planning threats, with values between 0 and 1 , was calculated by means of the following Equation (3):

$$
W_{I(I I)}=\frac{1}{n} \sum_{j=1}^{n} z_{i j}
$$

where: 
$W_{I}$-the synthetic indicator of the environmental-infrastructural threats to sustainable tourism in the municipalities;

$W_{I I}$-the synthetic indicator of the environmental-planning threats to sustainable tourism in the municipalities;

$j-1,2, \ldots, \mathrm{n}$;

$n$ - the number of characteristics taken into account.

In order to achieve impartial diversification of the municipalities, a decision was made to carry out standardization separately for rural and urban-rural municipalities. In the final stage, a division into typological groups was applied in accordance with a principle presented in the survey results.

In addition, the coefficient between the obtained sustainable tourism indicators (calculated as the average of environmental-infrastructural threats and environmental-planning threats indicators) and the tourist attractiveness indicators [7] was analysed. To estimate the correlation between the scoring results obtained for the two types of assessments, the statistical analysis was conducted by calculating the Pearson correlation coefficient (r).

Taking into account the research objectives and the adopted methodology, the following thesis was formulated: environmental-infrastructural and environmental-planning activities in municipalities with high nature and tourism attractiveness should be implemented very carefully and meticulously.

\section{Results}

A detailed set of calculated variables, the results of their normalization, and the values of the synthetic environmental-infrastructural $\left(\boldsymbol{W}_{I}\right)$ and environmental-planning $\left(\boldsymbol{W}_{I I}\right)$ indicators of sustainable tourism in the analysed municipalities are presented in Table 1. 
Table 1. Values of the variables, normalized indicators, and synthetic indicators of sustainable tourism in the analyzed municipalities (own elaboration).

\begin{tabular}{|c|c|c|c|c|c|c|c|c|c|c|c|c|c|c|c|c|c|c|}
\hline No. & Municipality & NP 1. & $X_{1}$ & $X_{2}$ & $X_{3}$ & $X_{4}$ & $X_{5}$ & $X_{6}$ & $X_{7}$ & $\mathrm{Z}_{1}$ & $\mathrm{Z}_{2}$ & $\mathrm{Z}_{3}$ & $\mathrm{Z}_{4}$ & $\mathrm{Z}_{5}$ & $\mathrm{Z}_{6}$ & $\mathrm{Z}_{7}$ & $W_{I}$ & $W_{I I}$ \\
\hline \multicolumn{19}{|c|}{ Rural municipalities $^{2}$} \\
\hline 1 & Górzyca & \multirow{2}{*}{ 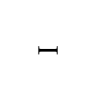 } & 0.033 & 0.201 & 0.281 & 3.941 & 0 & 12.03 & 9.833 & 0.746 & 0.412 & 0.719 & 0.932 & 1.000 & 0.258 & 0.117 & 0.626 & 0.577 \\
\hline 2 & Słońsk & & 0.028 & 0.222 & 0.232 & 0.359 & 0.008 & 6.38 & 16.491 & 0.785 & 0.351 & 0.768 & 0.994 & 0.999 & 0.137 & 0.196 & 0.634 & 0.582 \\
\hline 3 & Lipnica Wielka & \multirow{2}{*}{$\exists$} & 0.023 & 0.060 & 0.191 & 0 & 0 & 0.10 & 0.000 & 0.823 & 0.825 & 0.809 & 1.000 & 1.000 & 0.002 & 0.000 & 0.819 & 0.501 \\
\hline 4 & Zawoja & & 0.004 & 0.077 & 0.835 & 10.566 & 0 & 0.87 & 0.000 & 0.969 & 0.775 & 0.165 & 0.818 & 1.000 & 0.019 & 0.000 & 0.636 & 0.459 \\
\hline 5 & Narewka & \multirow[b]{2}{*}{$\Xi$} & 0.016 & 0.165 & 0.083 & 6.318 & 0.428 & 0.00 & 4.650 & 0.877 & 0.518 & 0.917 & 0.891 & 0.946 & 0.000 & 0.055 & 0.770 & 0.473 \\
\hline 6 & Białowieża & & 0.044 & 0.286 & 0.100 & 2.610 & 0.138 & 0.63 & 18.062 & 0.662 & 0.164 & 0.900 & 0.955 & 0.983 & 0.014 & 0.215 & 0.575 & 0.542 \\
\hline 7 & Wizna & \multirow{8}{*}{$\geq$} & 0.006 & 0.100 & 0.518 & 0 & 0.033 & 0.71 & 2.149 & 0.954 & 0.708 & 0.482 & 1.000 & 0.996 & 0.015 & 0.026 & 0.714 & 0.509 \\
\hline 8 & Nowy Dwór & & 0.005 & 0.074 & 0.178 & 0.109 & 0 & 0.00 & 84.025 & 0.962 & 0.784 & 0.822 & 0.998 & 1.000 & 0.000 & 1.000 & 0.856 & 0.750 \\
\hline 9 & Bargłów Kościelny & & 0.003 & 0.097 & 0.438 & 3.272 & 0 & 2.33 & 0.596 & 0.977 & 0.716 & 0.562 & 0.944 & 1.000 & 0.050 & 0.007 & 0.752 & 0.500 \\
\hline 10 & Grajewo & & 0.001 & 0.082 & 0.443 & 0.121 & 0.035 & 0.85 & 1.058 & 0.992 & 0.760 & 0.557 & 0.998 & 0.996 & 0.018 & 0.013 & 0.770 & 0.506 \\
\hline 11 & Jaświły & & 0.007 & 0.081 & 0.442 & 1.930 & 0.102 & 23.75 & 1.748 & 0.946 & 0.763 & 0.558 & 0.967 & 0.987 & 0.510 & 0.021 & 0.756 & 0.621 \\
\hline 12 & Radziłów & & 0.007 & 0.087 & 0.330 & 0 & 0 & 10.03 & 0.000 & 0.946 & 0.746 & 0.670 & 1.000 & 1.000 & 0.215 & 0.000 & 0.787 & 0.554 \\
\hline 13 & Sztabin & & 0.005 & 0.126 & 0.524 & 0.305 & 0 & 0.00 & 4.883 & 0.962 & 0.632 & 0.476 & 0.995 & 1.000 & 0.000 & 0.058 & 0.690 & 0.513 \\
\hline 14 & Trzcianne & & 0.008 & 0.104 & 0.370 & 0.462 & 0.058 & 0.00 & 0.000 & 0.938 & 0.696 & 0.630 & 0.992 & 0.993 & 0.000 & 0.000 & 0.755 & 0.496 \\
\hline 15 & Czarna & \multirow{3}{*}{$>$} & 0.004 & 0.078 & 0.752 & 0.981 & 0 & 0.96 & 1.895 & 0.969 & 0.772 & 0.248 & 0.983 & 1.000 & 0.021 & 0.023 & 0.663 & 0.507 \\
\hline 16 & Cisna & & 0.050 & 0.206 & 0.241 & 0.399 & 0 & 0.52 & 0.943 & 0.615 & 0.398 & 0.759 & 0.993 & 1.000 & 0.011 & 0.011 & 0.591 & 0.504 \\
\hline 17 & Lutowiska & & 0.014 & 0.082 & 0.169 & 0 & 0 & 2.72 & 3.105 & 0.892 & 0.760 & 0.831 & 1.000 & 1.000 & 0.058 & 0.037 & 0.828 & 0.524 \\
\hline 18 & Chojnice & 5 & 0.033 & 0.214 & 0.134 & 1.752 & 0 & 46.57 & 61.062 & 0.746 & 0.374 & 0.866 & 0.970 & 1.000 & 1.000 & 0.727 & 0.662 & 0.924 \\
\hline 19 & Bierzwnik & F & 0.011 & 0.130 & 0.419 & 0.004 & 0 & 11.24 & 0.000 & 0.915 & 0.620 & 0.581 & 1.000 & 1.000 & 0.241 & 0.000 & 0.705 & 0.560 \\
\hline 20 & Nowy Targ & \multirow{5}{*}{$\stackrel{\Xi}{>}$} & 0.011 & 0.083 & 0.291 & 0.108 & 0.023 & 0.80 & 0.063 & 0.915 & 0.757 & 0.709 & 0.998 & 0.997 & 0.017 & 0.001 & 0.794 & 0.503 \\
\hline 21 & Ochotnica Dolna & & 0.035 & 0.097 & 0.116 & 0 & 0 & 0.01 & 0.000 & 0.731 & 0.716 & 0.884 & 1.000 & 1.000 & 0.000 & 0.000 & 0.777 & 0.500 \\
\hline 22 & Mszana Dolna & & 0.011 & 0.053 & 0.582 & 0 & 0 & 0.00 & 0.000 & 0.915 & 0.845 & 0.418 & 1.000 & 1.000 & 0.000 & 0.000 & 0.726 & 0.500 \\
\hline 23 & Kamienica & & 0.009 & 0.067 & 0.183 & 0 & 0.014 & 0.00 & 0.476 & 0.931 & 0.804 & 0.817 & 1.000 & 0.998 & 0.000 & 0.006 & 0.851 & 0.501 \\
\hline 24 & Niedźwiedź & & 0.009 & 0.090 & 0.686 & 14.377 & 0.034 & 2.51 & 0.000 & 0.931 & 0.737 & 0.314 & 0.753 & 0.996 & 0.054 & 0.000 & 0.661 & 0.451 \\
\hline 25 & Lewin Kłodzki & $\breve{a}$ & 0.022 & 0.204 & 0.023 & 1.708 & 0 & 9.13 & 4.573 & 0.831 & 0.404 & 0.977 & 0.971 & 1.000 & 0.196 & 0.054 & 0.737 & 0.555 \\
\hline
\end{tabular}


Table 1. Cont

\begin{tabular}{|c|c|c|c|c|c|c|c|c|c|c|c|c|c|c|c|c|c|c|}
\hline No. & Municipality & NP 1. & $X_{1}$ & $X_{2}$ & $X_{3}$ & $X_{4}$ & $X_{5}$ & $x_{6}$ & $x_{7}$ & $\mathrm{Z}_{1}$ & $\mathrm{Z}_{2}$ & $\mathrm{Z}_{3}$ & $\mathrm{Z}_{4}$ & $\mathrm{Z}_{5}$ & $\mathrm{Z}_{6}$ & $\mathrm{Z}_{7}$ & $W_{I}$ & $W_{I I}$ \\
\hline \multicolumn{19}{|c|}{ Rural municipalities ${ }^{2}$} \\
\hline 26 & Tomaszów Maz. & \multirow{8}{*}{$\times$} & 0.004 & 0.208 & 0.686 & 3.141 & 0 & 4.51 & 5.524 & 0.969 & 0.392 & 0.314 & 0.946 & 1.000 & 0.097 & 0.066 & 0.558 & 0.527 \\
\hline 27 & Stare Babice & & 0.033 & 0.288 & 0.169 & 0 & 1.560 & 7.49 & 35.021 & 0.746 & 0.158 & 0.831 & 1.000 & 0.802 & 0.161 & 0.417 & 0.578 & 0.595 \\
\hline 28 & Kampinos & & 0.020 & 0.174 & 0.657 & 10.691 & 2.017 & 21.53 & 4.655 & 0.846 & 0.491 & 0.343 & 0.816 & 0.744 & 0.462 & 0.055 & 0.560 & 0.520 \\
\hline 29 & Brochów & & 0.020 & 0.158 & 0.328 & 38.960 & 0.407 & 0.00 & 6.946 & 0.846 & 0.538 & 0.672 & 0.330 & 0.948 & 0.000 & 0.083 & 0.685 & 0.340 \\
\hline 30 & Izabelin & & 0.053 & 0.244 & 0.235 & 12.471 & 0 & 37.10 & 3.137 & 0.592 & 0.287 & 0.765 & 0.785 & 1.000 & 0.797 & 0.037 & 0.548 & 0.655 \\
\hline 31 & Czosnów & & 0.017 & 0.197 & 0.197 & 1.058 & 0 & 1.62 & 0.449 & 0.869 & 0.424 & 0.803 & 0.982 & 1.000 & 0.035 & 0.005 & 0.699 & 0.505 \\
\hline 32 & Leszno & & 0.026 & 0.242 & 0.450 & 16.436 & 1.092 & 4.39 & 4.545 & 0.800 & 0.292 & 0.550 & 0.717 & 0.862 & 0.094 & 0.054 & 0.547 & 0.432 \\
\hline 33 & Leoncin & & 0.009 & 0.124 & 0.463 & 17.408 & 0.306 & 1.20 & 0.181 & 0.931 & 0.637 & 0.537 & 0.700 & 0.961 & 0.026 & 0.002 & 0.702 & 0.422 \\
\hline 34 & Podgórzyn & $\bar{x}$ & 0.036 & 0.232 & 0.336 & 5.479 & 0.288 & 0.00 & 1.162 & 0.723 & 0.322 & 0.664 & 0.906 & 0.963 & 0.000 & 0.014 & 0.570 & 0.471 \\
\hline 35 & Osiek Jasielski & \multirow{6}{*}{$\ddot{x}$} & 0.007 & 0.078 & 0.493 & 12.563 & 0.037 & 10.41 & 0.000 & 0.946 & 0.772 & 0.507 & 0.784 & 0.995 & 0.224 & 0.000 & 0.742 & 0.501 \\
\hline 36 & Sękowa & & 0.019 & 0.095 & 0.137 & 5.376 & 0.984 & 3.00 & 0.201 & 0.854 & 0.722 & 0.863 & 0.908 & 0.875 & 0.064 & 0.002 & 0.813 & 0.462 \\
\hline 37 & Lipinki & & 0.013 & 0.105 & 0.072 & 0 & 0 & 1.45 & 0.000 & 0.900 & 0.693 & 0.928 & 1.000 & 1.000 & 0.031 & 0.000 & 0.840 & 0.508 \\
\hline 38 & Nowy Żmigród & & 0.010 & 0.072 & 0.476 & 0 & 0 & 1.42 & 2.701 & 0.923 & 0.789 & 0.524 & 1.000 & 1.000 & 0.030 & 0.032 & 0.746 & 0.516 \\
\hline 39 & Dębowiec & & 0.014 & 0.084 & 0.746 & 0 & 0.113 & 0.02 & 0.000 & 0.892 & 0.754 & 0.254 & 1.000 & 0.986 & 0.000 & 0.000 & 0.634 & 0.497 \\
\hline 40 & Krempna & & 0.019 & 0.079 & 0.269 & 0 & 0 & 0.87 & 32.377 & 0.854 & 0.769 & 0.731 & 1.000 & 1.000 & 0.019 & 0.385 & 0.785 & 0.601 \\
\hline 41 & Kobylin-Borzymy & \multirow{3}{*}{ 貝 } & 0.000 & 0.056 & 0.680 & 1.468 & 0 & 0.00 & 0.000 & 1.000 & 0.836 & 0.320 & 0.975 & 1.000 & 0.000 & 0.000 & 0.719 & 0.494 \\
\hline 42 & Sokoły & & 0.007 & 0.095 & 0.515 & 0.821 & 0.048 & 0.00 & 0.120 & 0.946 & 0.722 & 0.485 & 0.986 & 0.994 & 0.000 & 0.001 & 0.718 & 0.495 \\
\hline 43 & Turośń Kościelna & & 0.009 & 0.092 & 0.454 & 0 & 0 & 0.00 & 0.000 & 0.931 & 0.731 & 0.546 & 1.000 & 1.000 & 0.000 & 0.000 & 0.736 & 0.500 \\
\hline 44 & Wielka Wieś & \multirow{3}{*}{$\vec{x}$} & 0.031 & 0.233 & 0.037 & 0.505 & 0 & 9.59 & 2.228 & 0.762 & 0.319 & 0.963 & 0.991 & 1.000 & 0.206 & 0.027 & 0.681 & 0.556 \\
\hline 45 & $\begin{array}{l}\text { Jerzmanowice } \\
\text {-Przeginia }\end{array}$ & & 0.013 & 0.130 & 0.670 & 0.221 & 0 & 0.51 & 0.000 & 0.900 & 0.620 & 0.330 & 0.996 & 1.000 & 0.011 & 0.000 & 0.617 & 0.502 \\
\hline 46 & Sułoszowa & & 0.018 & 0.101 & 0.149 & 0 & 0 & 0.00 & 0.000 & 0.862 & 0.705 & 0.851 & 1.000 & 1.000 & 0.000 & 0.000 & 0.806 & 0.500 \\
\hline 47 & Łapsze Niżne & \multirow{3}{*}{$\vec{x}$} & 0.019 & 0.090 & 0.043 & 0 & 0 & 6.92 & 4.459 & 0.854 & 0.737 & 0.957 & 1.000 & 1.000 & 0.149 & 0.053 & 0.849 & 0.550 \\
\hline 48 & $\begin{array}{l}\text { Krościenkon/ } \\
\text { Dunajcem }\end{array}$ & & 0.016 & 0.139 & 0.328 & 0.739 & 7.887 & 9.18 & 0.000 & 0.877 & 0.594 & 0.672 & 0.987 & 0.000 & 0.197 & 0.000 & 0.714 & 0.296 \\
\hline 49 & Czorsztyn & & 0.023 & 0.087 & 0.092 & 0 & 0 & 5.32 & 0.000 & 0.823 & 0.746 & 0.908 & 1.000 & 1.000 & 0.114 & 0.000 & 0.826 & 0.529 \\
\hline
\end{tabular}


Table 1. Cont

\begin{tabular}{|c|c|c|c|c|c|c|c|c|c|c|c|c|c|c|c|c|c|c|}
\hline No. & Municipality & NP 1. & $X_{1}$ & $X_{2}$ & $X_{3}$ & $X_{4}$ & $X_{5}$ & $X_{6}$ & $x_{7}$ & $\mathrm{Z}_{1}$ & $\mathrm{Z}_{2}$ & $\mathrm{Z}_{3}$ & $\mathrm{Z}_{4}$ & $\mathrm{Z}_{5}$ & $\mathrm{Z}_{6}$ & $\mathbf{Z}_{7}$ & $W_{I}$ & $W_{I I}$ \\
\hline \multicolumn{19}{|c|}{ Rural municipalities $^{2}$} \\
\hline 50 & Ludwin & \multirow{6}{*}{$\underset{x}{\Sigma}$} & 0.010 & 0.048 & 0.403 & 0.017 & 0 & 0.33 & 0.092 & 0.923 & 0.860 & 0.597 & 1.000 & 1.000 & 0.007 & 0.001 & 0.793 & 0.502 \\
\hline 51 & Stary Brus & & 0.008 & 0.048 & 0.102 & 2.646 & 0 & 8.35 & 7.545 & 0.938 & 0.860 & 0.898 & 0.954 & 1.000 & 0.179 & 0.090 & 0.899 & 0.556 \\
\hline 52 & Hańsk & & 0.016 & 0.066 & 0.038 & 0 & 0 & 2.90 & 5.406 & 0.877 & 0.807 & 0.962 & 1.000 & 1.000 & 0.062 & 0.064 & 0.882 & 0.532 \\
\hline 53 & Wierzbica & & 0.013 & 0.075 & 0.532 & 1.095 & 0 & 0.96 & 2.275 & 0.900 & 0.781 & 0.468 & 0.981 & 1.000 & 0.021 & 0.027 & 0.716 & 0.507 \\
\hline 54 & Sosnowica & & 0.015 & 0.058 & 0.409 & 0 & 0 & 1.20 & 3.692 & 0.885 & 0.830 & 0.591 & 1.000 & 1.000 & 0.026 & 0.044 & 0.769 & 0.517 \\
\hline 55 & Urszulin & & 0.012 & 0.098 & 0.417 & 3.553 & 0 & 0.00 & 0.145 & 0.908 & 0.713 & 0.583 & 0.939 & 1.000 & 0.000 & 0.002 & 0.735 & 0.485 \\
\hline 56 & Zamość & \multirow{2}{*}{$\underset{x}{P}$} & 0.006 & 0.092 & 0.288 & 7.286 & 0 & 0.00 & 1.114 & 0.992 & 0.839 & 0.409 & 1.000 & 1.000 & 0.000 & 0.000 & 0.747 & 0.500 \\
\hline 57 & Adamów & & 0.001 & 0.055 & 0.591 & 0 & 0 & 0.00 & 0.000 & 0.954 & 0.731 & 0.712 & 0.875 & 1.000 & 0.000 & 0.013 & 0.799 & 0.472 \\
\hline 58 & Ustka & \multirow{4}{*}{$\underset{x}{B}$} & 0.130 & 0.291 & 0.096 & 0 & 0 & 0.00 & 31.731 & 0.000 & 0.149 & 0.904 & 1.000 & 1.000 & 0.000 & 0.378 & 0.351 & 0.594 \\
\hline 59 & Główczyce & & 0.012 & 0.099 & 0.610 & 1.240 & 0 & 0.00 & 26.496 & 0.908 & 0.711 & 0.390 & 0.979 & 1.000 & 0.000 & 0.315 & 0.669 & 0.573 \\
\hline 60 & Wicko & & 0.023 & 0.175 & 0.133 & 0.247 & 0 & 9.99 & 0.657 & 0.823 & 0.488 & 0.867 & 0.996 & 1.000 & 0.215 & 0.008 & 0.726 & 0.555 \\
\hline 61 & Smołdzino & & 0.004 & 0.156 & 0.364 & 3.164 & 0 & 1.13 & 3.176 & 0.969 & 0.544 & 0.636 & 0.946 & 1.000 & 0.024 & 0.038 & 0.716 & 0.502 \\
\hline 62 & Górno & \multirow{5}{*}{$\stackrel{\grave{x}}{x}$} & 0.011 & 0.051 & 0.661 & 0 & 0 & 0.43 & 0.000 & 0.915 & 0.851 & 0.339 & 1.000 & 1.000 & 0.009 & 0.000 & 0.702 & 0.502 \\
\hline 63 & Masłów & & 0.016 & 0.072 & 0.292 & 15.089 & 0 & 0.00 & 1.030 & 0.877 & 0.789 & 0.708 & 0.740 & 1.000 & 0.000 & 0.012 & 0.791 & 0.438 \\
\hline 64 & Łączna & & 0.008 & 0.067 & 0.383 & 0.295 & 0.027 & 0.00 & 0.172 & 0.938 & 0.804 & 0.617 & 0.995 & 0.997 & 0.000 & 0.002 & 0.787 & 0.498 \\
\hline 65 & Bieliny & & 0.011 & 0.032 & 0.129 & 9.045 & 1.099 & 0.00 & 0.196 & 0.915 & 0.906 & 0.871 & 0.844 & 0.861 & 0.000 & 0.002 & 0.898 & 0.427 \\
\hline 66 & Nowa Słupia & & 0.011 & 0.000 & 0.209 & 0.806 & 1.141 & 1.72 & 2.798 & 0.915 & 1.000 & 0.791 & 0.986 & 0.855 & 0.037 & 0.033 & 0.902 & 0.478 \\
\hline 67 & Poronin & \multirow{3}{*}{$\stackrel{x}{x}$} & 0.025 & 0.190 & 0.516 & 1.667 & 0 & 0.00 & 0.000 & 0.808 & 0.444 & 0.484 & 0.971 & 1.000 & 0.000 & 0.000 & 0.579 & 0.493 \\
\hline 68 & Bukowina & & 0.023 & 0.191 & 0.800 & 1.647 & 0.015 & 2.93 & 0.000 & 0.823 & 0.442 & 0.200 & 0.972 & 0.998 & 0.063 & 0.000 & 0.488 & 0.508 \\
\hline 69 & Kościelisko & & 0.026 & 0.195 & 0.458 & 2.962 & 0.102 & 0.00 & 0.406 & 0.800 & 0.430 & 0.542 & 0.949 & 0.987 & 0.000 & 0.005 & 0.591 & 0.485 \\
\hline 70 & Dopiewo & \multirow{2}{*}{$\vec{x}$} & 0.032 & 0.328 & 0.137 & 9.594 & 0 & 22.98 & 4.342 & 0.754 & 0.041 & 0.863 & 0.835 & 1.000 & 0.493 & 0.052 & 0.553 & 0.595 \\
\hline 71 & Komorniki & & 0.045 & 0.342 & 0.054 & 0 & 0 & 17.82 & 4.584 & 0.654 & 0.000 & 0.946 & 1.000 & 1.000 & 0.383 & 0.055 & 0.533 & 0.609 \\
\hline 72 & Krasnopol & \multirow{4}{*}{$\stackrel{\bar{x}}{x}$} & 0.000 & 0.080 & 0.511 & 0 & 0 & 0.00 & 1.545 & 1.000 & 0.766 & 0.489 & 1.000 & 1.000 & 0.000 & 0.018 & 0.752 & 0.505 \\
\hline 73 & Nowinka & & 0.008 & 0.125 & 0.134 & 0 & 0.064 & 0.00 & 1.208 & 0.938 & 0.635 & 0.866 & 1.000 & 0.992 & 0.000 & 0.014 & 0.813 & 0.502 \\
\hline 74 & Giby & & 0.000 & 0.089 & 1.194 & 4.630 & 0.195 & 0.00 & 0.000 & 1.000 & 0.740 & 0.000 & 0.920 & 0.975 & 0.000 & 0.000 & 0.580 & 0.474 \\
\hline 75 & Suwałki & & 0.012 & 0.114 & 0.245 & 58.121 & 0.112 & 0.12 & 5.468 & 0.908 & 0.667 & 0.755 & 0.000 & 0.986 & 0.003 & 0.065 & 0.776 & 0.263 \\
\hline
\end{tabular}


Table 1. Cont.

\begin{tabular}{|c|c|c|c|c|c|c|c|c|c|c|c|c|c|c|c|c|c|c|}
\hline No. & Municipality & NP 1. & $x_{1}$ & $\mathrm{X}_{2}$ & $x_{3}$ & $X_{4}$ & $X_{5}$ & $X_{6}$ & $x_{7}$ & $\mathrm{Z}_{1}$ & $\mathrm{Z}_{2}$ & $\mathrm{Z}_{3}$ & $\mathrm{Z}_{4}$ & $\mathrm{Z}_{5}$ & $\mathrm{Z}_{6}$ & $\mathrm{Z}_{7}$ & $W_{I}$ & $W_{I I}$ \\
\hline \multicolumn{19}{|c|}{ Urban-rural municipalities ${ }^{2}$} \\
\hline 76 & Witnica & 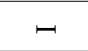 & 0.023 & 0.226 & 0.426 & 0 & 1.904 & 8.05 & 31.042 & 0.701 & 0.549 & 0.184 & 1.000 & 0.000 & 0.063 & 0.381 & 0.478 & 0.361 \\
\hline 77 & Jedwabne & \multirow{6}{*}{$\geq$} & 0.006 & 0.140 & 0.380 & 0.445 & 0 & 0.73 & 6.936 & 0.922 & 0.721 & 0.272 & 0.996 & 1.000 & 0.006 & 0.085 & 0.638 & 0.522 \\
\hline 78 & Rajgród & & 0.016 & 0.189 & 0.417 & 0.355 & 0.158 & 0.62 & 18.591 & 0.792 & 0.623 & 0.201 & 0.996 & 0.917 & 0.005 & 0.228 & 0.539 & 0.537 \\
\hline 79 & Lipsk & & 0.010 & 0.141 & 0.340 & 1.493 & 0.136 & 8.13 & 6.968 & 0.870 & 0.719 & 0.349 & 0.985 & 0.929 & 0.064 & 0.086 & 0.646 & 0.516 \\
\hline 80 & $\begin{array}{c}\text { Dabrowa } \\
\text { Białostocka }\end{array}$ & & 0.034 & 0.118 & 0.126 & 0.257 & 0 & 24.49 & 4.978 & 0.558 & 0.764 & 0.759 & 0.997 & 1.000 & 0.192 & 0.061 & 0.694 & 0.563 \\
\hline 81 & Suchowola & & 0.010 & 0.087 & 0.186 & 0.020 & 0 & 11.17 & 4.942 & 0.870 & 0.826 & 0.644 & 1.000 & 1.000 & 0.088 & 0.061 & 0.780 & 0.537 \\
\hline 82 & Goniądz & & 0.005 & 0.201 & 0.478 & 0.007 & 0 & 3.20 & 11.272 & 0.935 & 0.599 & 0.084 & 1.000 & 1.000 & 0.025 & 0.138 & 0.539 & 0.541 \\
\hline 83 & Krzyż Wlp. & \multirow{5}{*}{$\xi$} & 0.021 & 0.229 & 0.456 & 0.336 & 0 & 5.56 & 13.289 & 0.727 & 0.543 & 0.126 & 0.997 & 1.000 & 0.044 & 0.163 & 0.466 & 0.551 \\
\hline 84 & Tuczno & & 0.020 & 0.190 & 0.340 & 0.568 & 0.029 & 6.71 & 4.738 & 0.740 & 0.621 & 0.349 & 0.994 & 0.985 & 0.053 & 0.058 & 0.570 & 0.522 \\
\hline 85 & Drawno & & 0.021 & 0.182 & 0.272 & 0 & 0 & 12.51 & 81.399 & 0.727 & 0.637 & 0.479 & 1.000 & 1.000 & 0.098 & 1.000 & 0.614 & 0.775 \\
\hline 86 & Człopa & & 0.015 & 0.180 & 0.251 & 0 & 0 & 7.91 & 25.005 & 0.805 & 0.641 & 0.519 & 1.000 & 1.000 & 0.062 & 0.307 & 0.655 & 0.592 \\
\hline 87 & Dobiegniew & & 0.021 & 0.339 & 0.126 & 0.630 & 0 & 11.56 & 11.164 & 0.727 & 0.323 & 0.759 & 0.994 & 1.000 & 0.091 & 0.137 & 0.603 & 0.555 \\
\hline 88 & Szczytna & \multirow{2}{*}{ 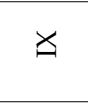 } & 0.013 & 0.156 & 0.375 & 0 & 0 & 9.10 & 1.335 & 0.831 & 0.689 & 0.282 & 1.000 & 1.000 & 0.071 & 0.016 & 0.600 & 0.522 \\
\hline 89 & Radków & & 0.017 & 0.196 & 0.137 & 0.608 & 0 & 0.82 & 27.038 & 0.779 & 0.609 & 0.738 & 0.994 & 1.000 & 0.006 & 0.332 & 0.709 & 0.583 \\
\hline 90 & Łomianki & $\times$ & 0.038 & 0.289 & 0.522 & 6.477 & 0.334 & 12.62 & 10.913 & 0.506 & 0.423 & 0.000 & 0.935 & 0.825 & 0.099 & 0.134 & 0.310 & 0.498 \\
\hline 91 & Tykocin & $\bar{x}$ & 0.018 & 0.153 & 0.274 & 100 & 0.523 & 1.92 & 1.719 & 0.766 & 0.695 & 0.475 & 0.000 & 0.725 & 0.015 & 0.021 & 0.645 & 0.190 \\
\hline 92 & Suraż & \multirow{3}{*}{ 貝 } & 0.008 & 0.128 & 0.123 & 0 & 0 & 0.00 & 2.959 & 0.896 & 0.745 & 0.764 & 1.000 & 1.000 & 0.000 & 0.036 & 0.802 & 0.509 \\
\hline 93 & Choroszcz & & 0.017 & 0.179 & 0.506 & 0.201 & 0 & 0.35 & 1.135 & 0.779 & 0.643 & 0.031 & 0.998 & 1.000 & 0.003 & 0.014 & 0.484 & 0.504 \\
\hline 94 & Łapy & & 0.029 & 0.196 & 0.059 & 0 & 0 & 2.24 & 30.620 & 0.623 & 0.609 & 0.887 & 1.000 & 1.000 & 0.018 & 0.376 & 0.706 & 0.598 \\
\hline 95 & Skała & $\vec{x}$ & 0.019 & 0.209 & 0.221 & 0.845 & 0.132 & 1.96 & 8.130 & 0.753 & 0.583 & 0.577 & 0.992 & 0.931 & 0.015 & 0.100 & 0.638 & 0.509 \\
\hline 96 & Szczawnica & $\vec{x}$ & 0.031 & 0.132 & 0.188 & 0 & 0 & 48.76 & 7.899 & 0.597 & 0.737 & 0.640 & 1.000 & 1.000 & 0.383 & 0.097 & 0.658 & 0.620 \\
\hline 97 & Józefów & \multirow{2}{*}{$\stackrel{8}{x}$} & 0.008 & 0.042 & 0.443 & 3.702 & 0.240 & 4.54 & 2.160 & 0.896 & 0.916 & 0.151 & 0.963 & 0.874 & 0.036 & 0.027 & 0.655 & 0.475 \\
\hline 98 & Zwierzyniec & & 0.027 & 0.113 & 0.286 & 0.080 & 0 & 13.36 & 13.783 & 0.649 & 0.774 & 0.452 & 0.999 & 1.000 & 0.105 & 0.169 & 0.625 & 0.568 \\
\hline 99 & Bodzentyn & $\stackrel{x}{x}$ & 0.011 & 0.059 & 0.364 & 0.490 & 0 & 1.79 & 0.162 & 0.857 & 0.882 & 0.303 & 0.995 & 1.000 & 0.014 & 0.002 & 0.681 & 0.503 \\
\hline
\end{tabular}


Table 1. Cont.

\begin{tabular}{|c|c|c|c|c|c|c|c|c|c|c|c|c|c|c|c|c|c|c|}
\hline No. & Municipality & NP 1. & $x_{1}$ & $\mathrm{X}_{2}$ & $X_{3}$ & $X_{4}$ & $X_{5}$ & $X_{6}$ & $x_{7}$ & $\mathrm{Z}_{1}$ & $\mathrm{Z}_{2}$ & $\mathrm{Z}_{3}$ & $\mathrm{Z}_{4}$ & $\mathrm{Z}_{5}$ & $\mathrm{Z}_{6}$ & $\mathrm{Z}_{7}$ & $W_{I}$ & $W_{I I}$ \\
\hline \multicolumn{19}{|c|}{ Urban-rural municipalities ${ }^{2}$} \\
\hline 100 & Mosina & \multirow{2}{*}{$\underset{x}{x}$} & 0.020 & 0.284 & 0.420 & 16.696 & 0.057 & 18.32 & 12.022 & 0.740 & 0.433 & 0.195 & 0.833 & 0.970 & 0.144 & 0.148 & 0.456 & 0.524 \\
\hline 101 & Stęszew & & 0.030 & 0.259 & 0.385 & 0.243 & 0 & 6.66 & 17.175 & 0.610 & 0.483 & 0.262 & 0.998 & 1.000 & 0.052 & 0.211 & 0.452 & 0.565 \\
\hline 102 & Wolin & \multirow{2}{*}{$\underset{8}{8}$} & 0.018 & 0.225 & 0.411 & 0 & 0 & 5.64 & 17.014 & 0.766 & 0.551 & 0.213 & 1.000 & 1.000 & 0.044 & 0.209 & 0.510 & 0.563 \\
\hline 103 & Międzyzdroje & & 0.077 & 0.501 & 0.046 & 0 & 0 & 127.43 & 18.004 & 0.000 & 0.000 & 0.912 & 1.000 & 1.000 & 1.000 & 0.221 & 0.304 & 0.805 \\
\hline
\end{tabular}

${ }^{1}$ I—Ujście Warty NP, II—Babia Góra NP, III—Białowieża NP, IV—Biebrza NP, V-Bieszczady NP VI—Bory Tucholskie NP, VII—Drawa NP, VIII-Gorce NP, IX-Góry Stołowe NP, X-Kampinos NP, XI—Karkonosze NP, XII-Magura NP, XIII-Narew NP, XIV—Ojców NP, XV—Pieniny NP, XVI—Polesie NP, XVII-Roztocze NP, XVIII—Słowiński NP, XIX—Świetokrzyski NP, XX-Tatra NP, XXI—Wielkopolska NP, XXII—Wigry NP, XXIII—Wolin NP. ${ }^{2}$ The municipality is the basic unit of administrative division in Poland. It encompasses either a city (urban municipality) or only a rural area (rural municipality) as well as a city with the surrounding rural area (urban-rural municipality). Such division is in accordance with the regulation of the Polish Council of Ministers resulting from the legal act of 8 March 1990 regarding local government (plain text-Dz. U. 2018 pos. 994 ); according to the methodology, urban municipalities were excluded from the study. 
In order to present the spatial distribution of the analysed municipalities according to the obtained values of the $W_{I}$ and $W_{I I}$ synthetic indicators, the administrative units were divided into five groups and five types (Figures 1 and 2). They reflect the level of activities regarding green areas, sewage and wastewater policy. A division into equal ranges was adopted as follows:

- $\quad$ municipalities at a very high level, where $\boldsymbol{W}_{I}>0.8$-type A; $\boldsymbol{W}_{I I}>0.8$-Group 1;

- municipalities at a high level, where $W_{I}\left(0.60 \div 0.80>\right.$-type $B ; W_{I I}(0.60 \div 0.80>$-Group 2;

- municipalities at a medium level, where $W_{I}\left(0.40 \div 0.60>\right.$-type $C ; W_{I I}(0.40 \div 0.60>$-Group 3 ;

- $\quad$ municipalities at a low-level, where $W_{I}\left(0.20 \div 0.40>\right.$-type D; $W_{I I}(0.20 \div 0.40>$-Group 4 ;

- municipalities at a very low level, where $W_{I}<0.2$-type E; $W_{I I}<0.2$ Group 5 .

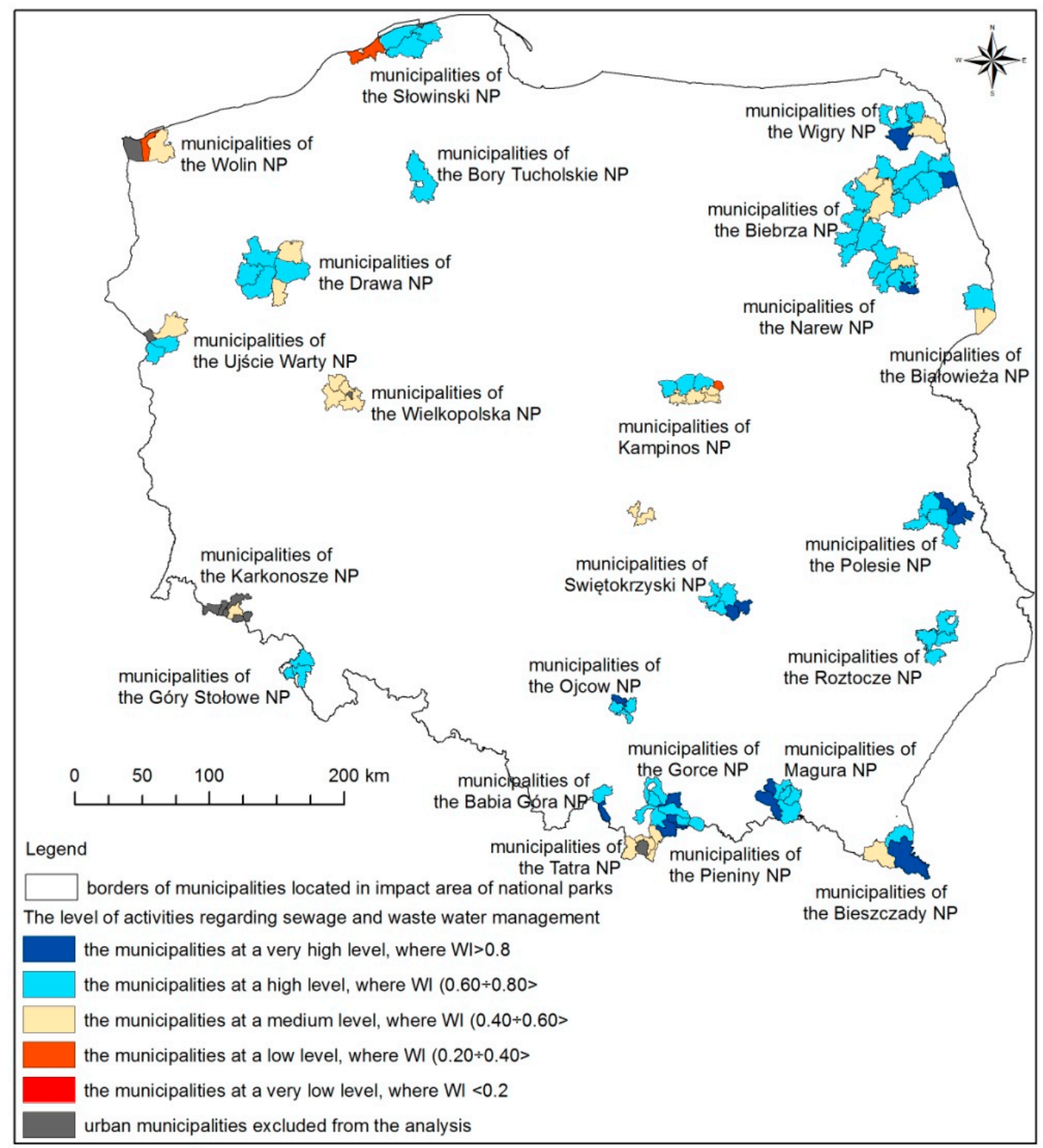

Figure 1. Spatial distribution of the municipality types classified by the synthetic environmentalinfrastructural indicator $W_{I}$ (own elaboration performed in ArcGIS). 


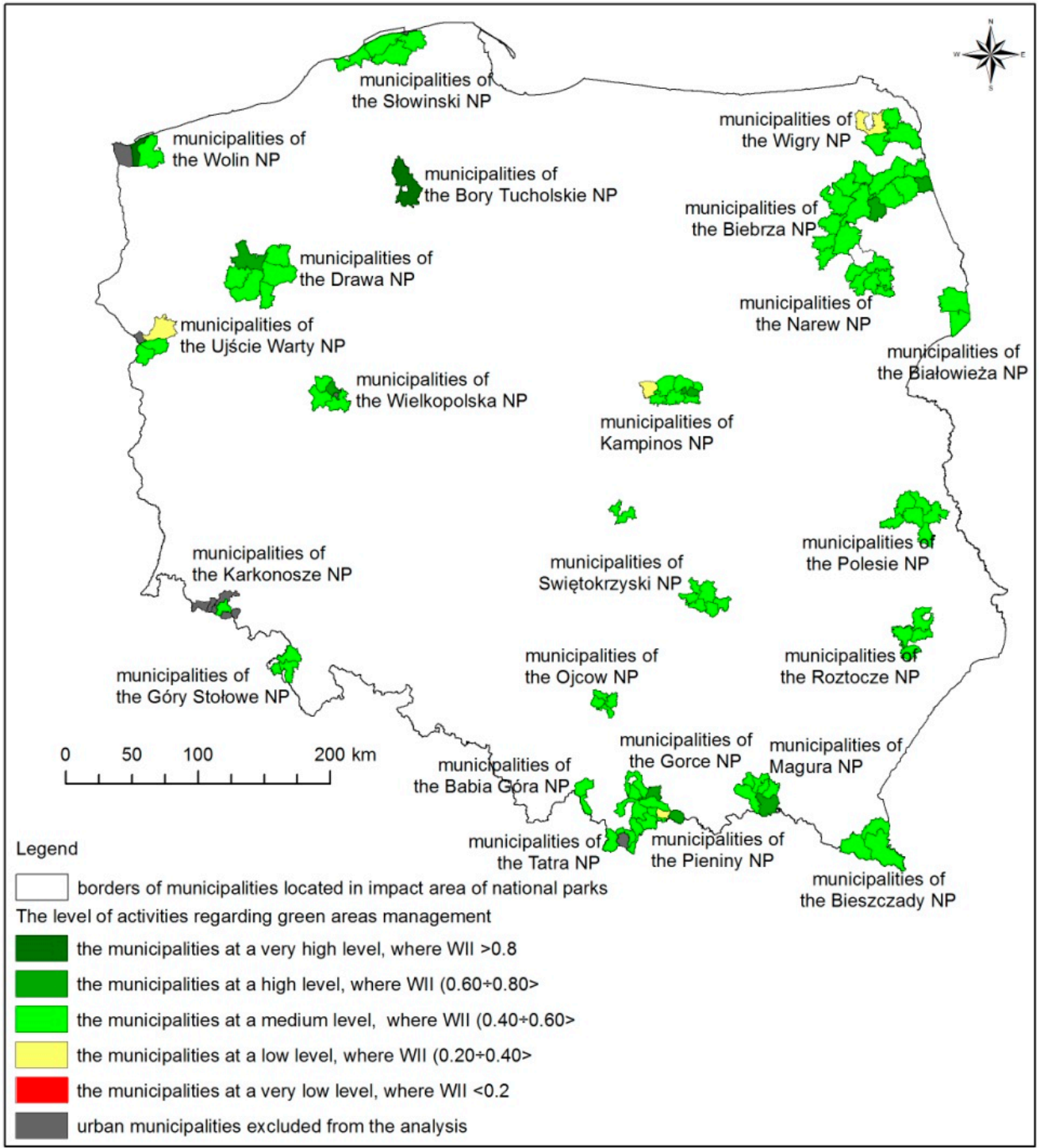

Figure 2. Spatial distribution of the municipality types classified by the synthetic environmentalplanning indicator $\boldsymbol{W}_{I I}$ (own elaboration).

The proposed division allowed for developing the typology of the municipalities. Table 2 illustrates the quantitative distribution of the municipalities with their particular characteristics.

Table 2. The quantitative distribution of the groups and types of municipalities related to the level of activities regarding green areas as well as sewage and wastewater policy.

\begin{tabular}{|c|c|c|c|c|c|c|c|}
\hline \multirow[t]{2}{*}{$\begin{array}{l}\text { Classification } \\
\text { of the Municipalities }\end{array}$} & & \multicolumn{5}{|c|}{$\begin{array}{l}\text { No. of the Municipalities Assigned to Specific Types } \\
\text { according to the Value of the } W_{I} \text { Indicator }\end{array}$} & \multirow{2}{*}{$\begin{array}{l}\text { No. of the Municipality } \\
\text { Types in Terms of Tourism } \\
\text { Sustainability } \\
\Sigma\end{array}$} \\
\hline & & $\mathbf{A}$ & B & $\mathrm{C}$ & D & E & \\
\hline \multirow{5}{*}{$\begin{array}{l}\text { No. of the } \\
\text { municipalities assigned } \\
\text { to specific groups } \\
\text { according to the value of } \\
\text { the } W_{I I} \text { indicator }\end{array}$} & 1 & $0^{1 .}$ & 1 & 0 & 1 & 0 & 2 \\
\hline & 2 & 2 & 4 & 2 & 0 & 0 & 8 \\
\hline & 3 & 13 & 53 & 20 & 2 & 0 & 88 \\
\hline & 4 & 0 & 3 & 1 & 0 & 0 & 4 \\
\hline & 5 & 0 & 1 & 0 & 0 & 0 & 1 \\
\hline$\Sigma$ & & 15 & 62 & 23 & 3 & 0 & 103 \\
\hline
\end{tabular}

1. The colors of the cells correspond to the indications set out in Figure 3. 
According to Table 2 presented above, five main categories of administrative units were distinguished (Figure 3).

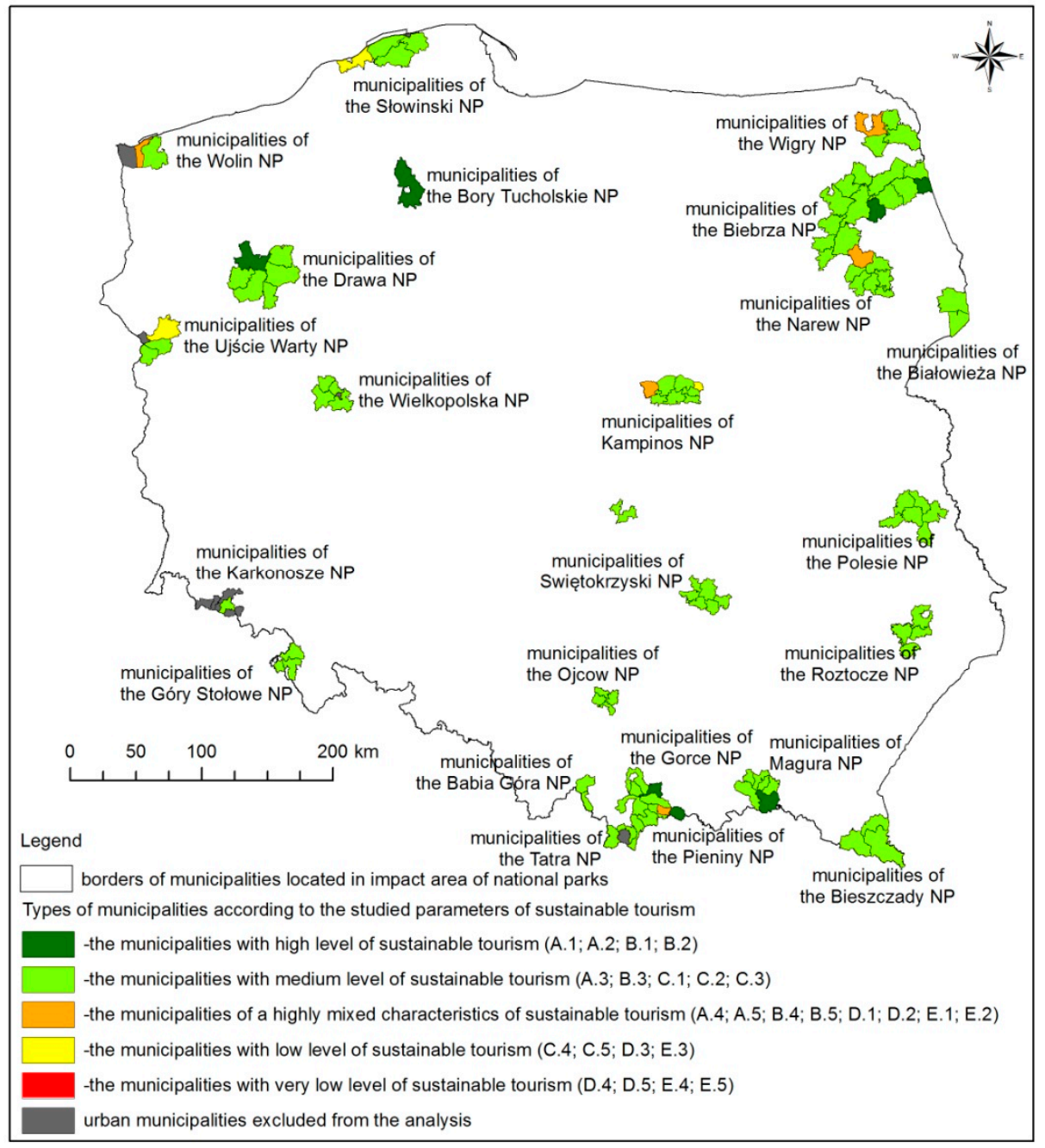

Figure 3. Spatial distribution of municipality types in terms of tourism sustainability.

The largest set of the municipalities (85\%) is the one with an average level of the studied parameters of sustainable tourism (type-group A-3, B-3, C-1, C-2, and C-3). These are the units where the development of water and sewage infrastructure, preservation of forest and agricultural landscape, and care for public green areas are carried out at the basic level. The second category $(7 \%)$ includes municipalities with a high degree of sustainable tourism (A-1, A-2, B-1, and B-2), in which the above activities were highly rated.

There are also very few extreme cases, which include:

- municipalities of highly mixed characteristics of sustainable tourism, where development in the field of sewage and waste infrastructure was evaluated very well, but activities related to the maintenance of green areas and preservation of forest-agricultural landscapes were evaluated negatively (A-4, A-5, B-4, and B-5)—4\%; or vice versa (D-1, D-2, E-1, and E-2)—1\%;

- municipalities with a low degree of the studied criteria of sustainable tourism, where infrastructural issues were evaluated at a medium level, but those related to green areas and forest-agricultural landscapes were rated very low (C-4 and C-5) $-1 \%$; or vice versa (D-3 and E-3) $-2 \%$.

Nevertheless, it is positive that no administrative units were found that rank very negatively in terms of all the studied criteria of sustainable tourism (D-4, D-5, E-4, and E-5); i.e., municipalities that 
carry out very limited activities, both in the field of wastewater and waste management, and are very inefficient in the preservation and protection of forest and agricultural landscapes, as well as in the maintenance and development of public green areas.

The study analyses also included the relation between the activities that affect the ecological aspect of sustainable tourism of the studied administrative units and their tourist attractiveness. The latter is presented in Figure 4.

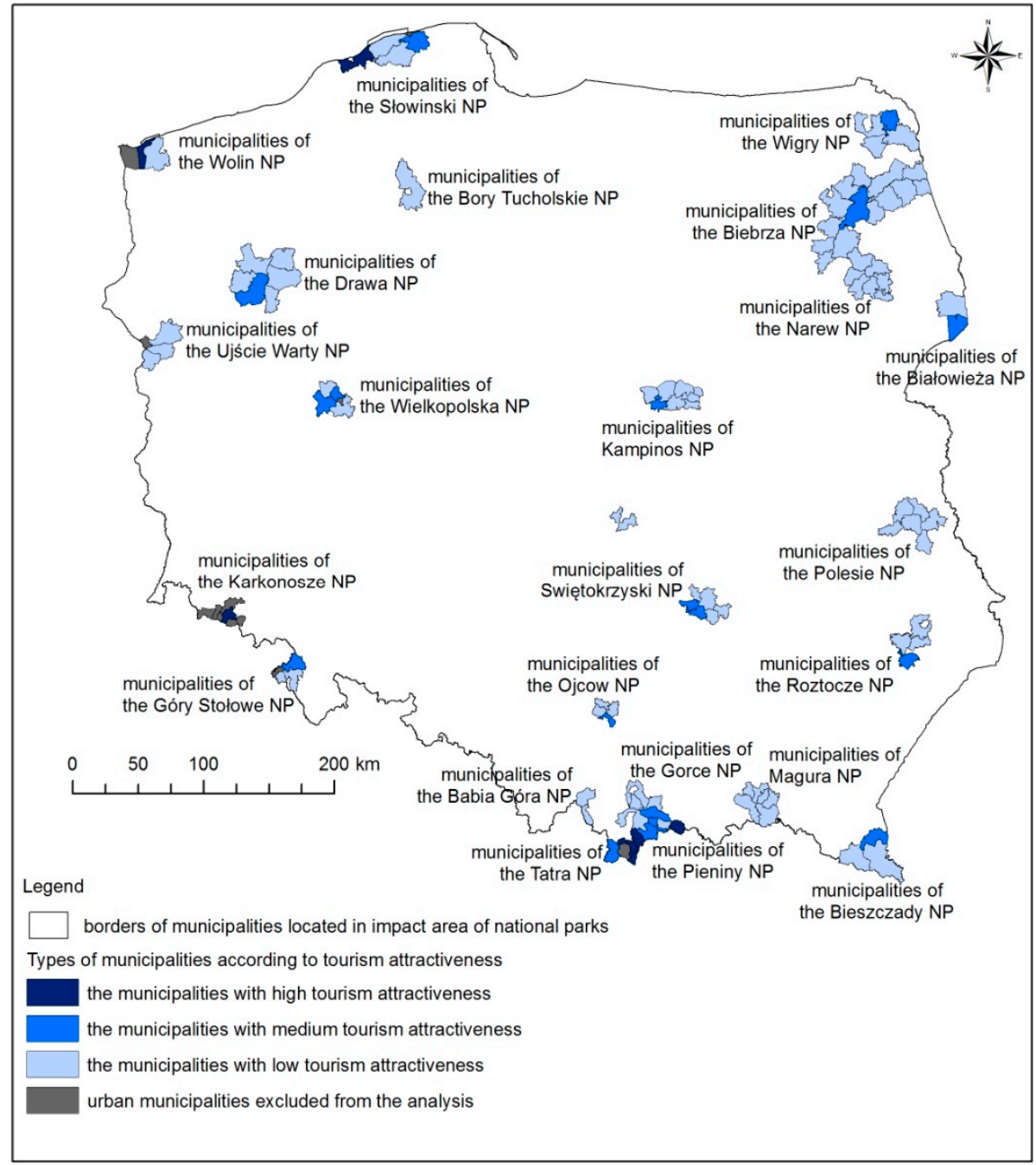

Figure 4. Spatial distribution of the municipality types in terms of tourism attractiveness [7].

Pearson's coefficient between the sustainable tourism indicators and the tourist attractiveness indicators was $r=-0.359$, signifying that the correlation is weak. The negative value suggest that the intensity of tourist traffic and the use of tourist accommodation is not highly related to the decision-making practices in the fields of municipal waste and water infrastructure or public greenery. For instance, the sea coastal municipalities, such as Ustka or Miedzyzdroje, are characterized by high tourist attractiveness along with a low or unstable level of sustainable tourism in terms of infrastructure and environmental planning. There are also reverse examples, e.g., the municipalities of Kamienica (in Narew NP) and Chojnice (Bory Tucholskie NP), with a high level of sustainable tourism but low tourist attractiveness. An outstanding example is the Szczawnica municipality (in Pieniny NP), which exhibits high tourist attractiveness along with a high level of sustainable tourism. 


\section{Discussion and Conclusions}

Several conclusions can be drawn on the basis of the analysis carried out above:

- the activities in the field of wastewater and waste management can be highly rated in 77 cases, medium in 23 cases, and very low in the case of 3 municipalities;

- the activities in the field of forest and rural landscape preservation as well as in the field of development and maintenance of green areas can be highly rated in the case of 10 municipalities, medium in 88 units, and low in 5 cases;

- the spatial diversification of the municipalities in terms of environmental and planning issues is low, but higher in terms of wastewater and waste management;

- the level of activities in the field of sewage and waste management, preservation of the forest and the agricultural landscape, and management of green areas is not related to a location in the impact area of a particular national park.

Low and medium scores for the indicators describing environmental and infrastructural threats to sustainable tourism may result, among others, from the lack of coordination between the development of new housing and tourism settlements and development of technical infrastructure. A bad practice often applied by local authorities is a high number of building permits issued for residential buildings, hotels, motels, and guest houses along with the scarcity of decisions taken on development of new technical infrastructure. Examples of such spatial management, according to the data for the years 2010-2018, are from two administrative units: Ustka Municipality, where 790 decisions on development conditions were issued and a $31.8 \mathrm{~km}$ sewage network was built; and Miedzyzdroje municipality, with 726 location decisions given in relation to a $4.2 \mathrm{~km}$ new sewage network. There is also the issue of the poor technical condition of the existing sewage network. Looking once again at the example of Ustka during the same period, the municipality suffered 1272 sewerage network failures.

The studied municipalities face the common process of a changing farmland status or its deforestation. The analysis of municipal spatial planning documents in Poland, concerning land-use changes and especially land designated for development (residential, service-provision, and tourist facilities), shows that local spatial development plans are seldom being developed to protect areas with biological and agricultural functions [34,39]. They are not an effective tool for organizing the spatial structure of urbanized areas. Instead, they rather concentrate on the development of new investment areas. One of the consequences of such a situation is the dispersion of settlements in rural areas and high costs of development of appropriate technical infrastructure.

Decisions concerning changes in land use for non-agricultural and non-forest purposes in municipalities with high natural value should be particularly well thought out, balanced, and limited to the essential minimum. However, in many cases, local authorities and inhabitants see greater economic benefits in the development of tourism than in the maintenance of the agricultural function and preservation of the agricultural and forest landscape. Examples of such objectionable practices include Ustka municipality (in Słowiński NP), where 74 ha of forest out of 6732 ha were repurposed in local development plans, as well as Łapy municipality (in Narew NP), with 137 ha out of 12,183 ha of forest designated for other purposes.

It is evident that the intensity of changes in the use of agricultural land for other purposes is much higher. In this case, two municipalities provide us with negative examples of such practice: Stare Babice and Czosnów, both located in the Kampinos NP National Park. In the former municipality, 1731 ha out of 4443 ha of agricultural land were repurposed, whereas in the latter one, 1240 ha out of 7123 ha. At the same time, 1102 ha of agricultural land out of 12,183 ha were changed for other purposes in Ustka municipality. It should be mentioned that, in some cases, the authorities of the national park have the obligation or right to issue opinions on land-use changes and location decisions for new development.

The indicator describing the maintenance and development of green areas appears to be related to the status of municipalities and their economic situation. The set of municipalities under study 
represents mainly rural and rural-urban municipalities. These are the administrative units which are probably less able to invest in the revitalization and development of public areas. This aspect was not included in the study.

The obtained typology of the municipalities leads to the conclusion that the hypothesis stated in the paper is true only in some cases. There are municipalities that carry out activities in the field of forest and agricultural land protection, invest in the maintenance and development of public green areas, properly manage sewage and wastewater, but at the same time are not attractive for tourists. This may be, among others, due to the effect of the lack of or insufficient preparation of accommodation resources. Examples of such municipalities include Chojnice and Kamienica, with low tourism attractiveness, but which were highly rated in terms of the evaluated criteria of sustainable tourism. Paradoxically, these units are in a better situation than municipalities where the situation is reversed. This refers to municipalities that are prime tourist destinations and have highly developed tourist accommodation and facilities, but do not keep up with sustainable tourism activities. This means high environmental costs of tourism in these areas. Such municipalities include Ustka and Międzyzdroje.

A positive example is the Szczawnica municipality, which was highly rated in terms of both the activities related to sustainable tourism and to tourist attractiveness. It serves as an example proving that it is possible to properly conduct environmentally friendly policy and allow significant development of tourism at the same time. However, this is a special case because the Szczawnica municipality is also a popular health resort. The local authorities therefore have to face additional formal obligations, mostly related to the protection of natural, landscape, and cultural values. At the same time, the spatial policy states several priorities that are strictly related to the concept of sustainable development. The authorities fulfil environmental, economic and social, spa and tourist, as well as cultural and sport tasks and demands. Such a multidimensional balanced spatial policy has been implemented in a comprehensive way. The municipal infrastructure includes services for all residents and tourists. A very rich and varied program of leisure and recreation infrastructure is implemented while maintaining the natural values of the Pieniny NP National Park and its buffer zone. Comprehensive revitalization is being carried out, not only of buildings and public spaces in the city, but also in rural areas [40].

This analysis led to the conclusion that, in the case of the majority of municipalities, the proposed evaluation criteria are to a lesser extent related to external tourist flow. They are more likely to properly assess the level of fulfilment of the permanent residents' needs. On the other hand, in some cases it can be proven that the applied criteria can be useful for assessment, especially in the case of municipalities with high tourism attractiveness.

Nevertheless, one may claim that in rural and urban-rural municipalities located in the impact area of national parks, environmentally friendly activities are mainly related to the spatial and infrastructural development of a municipality and the ecological awareness of its inhabitants and authorities. This means that the need to implement sustainable tourism objectives is not classified as being of utmost priority.

Author Contributions: Conceptualization, A.P.-Z. and K.P.; methodology, A.P.-Z. and K.P.; software, A.P.-Z. and K.P.; validation, A.P.-Z. and K.P.; formal analysis, A.P.-Z. and K.P.; investigation, A.P.-Z. and K.P.; resources, A.P.-Z. and K.P.; data curation, A.P.-Z. and K.P.; writing-original draft preparation, A.P.-Z. and K.P.; writing-review and editing, A.P.-Z.; visualization, K.P.; supervision, A.P.-Z.; project administration, A.P.-Z.; All authors have read and agreed to the published version of the manuscript.

Funding: This research received no external funding.

Conflicts of Interest: The authors declare no conflict of interest.

\section{References}

1. Environment 2019. Available online: https://stat.gov.pl/en/topics/environment-energy/environment/ environment-2019,1,11.html (accessed on 20 February 2020). 
2. Liszewski, S. Przestrzeń turystyczna parków narodowych w Polsce. In Gospodarka I Przestrzeń; Domański, B., Kurek, W., Eds.; IGiGP UJ: Kraków, Poland, 2009; pp. 187-201. ISBN 978-83-88424-45-8.

3. Zawilińska, B.; Mika, M. National parks and local development in Poland: A municipal perspective. Hum. Geogr. 2013, 7, 43-52. [CrossRef]

4. Schägner, J.P.; Brander, L.; Maes, J.; Paracchini, M.L.; Hartje, V. Mapping recreational visits and values of European National Parks by combining statistical modelling and unit value transfer. J. Nat. Conserv. 2016, 31, 71-84. [CrossRef]

5. Ziarnicka-Wojtaszek, A.; Zawora, T. Selected methods pertaining to the assessment of agricultural tourism attractiveness of rural regions. Infrastruktura i Ekologia Terenów Wiejskich 2011, 2011/02, 235-245.

6. Stokłosa, Ł.; Krupa, J. Sustainable tourism development in protected areas. Wyższa Szkoła Turystyki i Języków Obcych 2013, 11, 17-32.

7. Podawca, K.; Pawłat-Zawrzykraj, A. Diversifying tourism in municipalities within impact areas of national parks. Pol. J. Environ. Stud. 2018, 27, 2213-2227. [CrossRef]

8. Balmford, A.; Green, J.M.H.; Anderson, M.; Beresford, J.; Huang, C.; Naidoo, R.; Walpole, M.; Manica, A. Walk on the Wild Side: Estimating the Global Magnitude of Visits to Protected Areas. PLoS Biol. 2015, 13, e1002074. [CrossRef]

9. Simmonds, C.; Canon, G.; Wilkinson, T. Crisis in our national parks: How tourists are loving nature to death. Guard 2018. Available online: https:/www.theguardian.com/environment/2018/nov/20/nationalparks-america-overcrowding-crisis-tourism-visitation-solutions (accessed on 6 March 2020).

10. Eagles, P.F.J.; McCool, S.F. Tourism in National Parks and Protected Areas: Planning and Management; CABI Publishing: Oxfordshire, UK, 2002; ISBN 0-85199-589-6.

11. Puhakka, R. Increasing role of tourism in Finnish national parks. FENNIN-Int. J. Geogr. 2008, 186, 47-58.

12. Leung, Y.F.; Spenceley, A.; Hvenegaard, G.; Buckley, R. Tourism and Visitor Management in Protected Areas: Guidelines for Sustainability; Groves, C., Ed.; Best Practice Protected Area Guidelines Series; IUCN: Gland, Switzerland, 2018; ISBN 978-2-8317-1898-9.

13. IUCN. Commission on National Parks and Protected Areas. In Parks for Life: Action for Protected Areas in Europe; IUCN: Gland, Switzerland, 1994; ISBN 2-8317-0230-5.

14. Mitura, T. The rural tourism as the form of the sustainable tourism on the example of the podkarpacki province. In Europa w Ujęciu Interdyscyplinarnym—Społeczeństwo, Polityka, Gospodarka, Turystyka; Petrecka, B., Dyrda-Maciałek, S., Rejman, K., Eds.; Wydawnictwo Państwowej Wyższej Szkoły Techniczno-Ekonomicznej im. ks. Bronisława Markiewicza: Jarosław, Poland, 2015; pp. 337-350. ISBN 978-83-63909-71-0.

15. Kieżel, M.; Piotrowski, P.; Wiechoczek, J. The Research on Sustainable Tourism in the Light of Its Paradigms. Sustainability 2019, 11, 5821. [CrossRef]

16. Zaręba, D. Ekoturystyka, 3rd ed.; PWN: Warszawa, Poland, 2019; ISBN 978-83-01-16017-3.

17. WTO. Indicators of Sustainable Development for Tourism Destinations; A Guidebook World Tourism Organization; WTO: Madrid, Spain, 2004; ISBN 92-844-0726-5.

18. Oleśniewicz, P.; Pytel, S.; Markiewicz-Patkowska, J.; Szromek, A.R.; Jandová, S. A Model of the Sustainable Management of the Natural Environment in National Parks-A Case Study of National Parks in Poland. Sustainability 2020, 12, 2704. [CrossRef]

19. Weaver, D.B.; Lawton, L.J. A new visitation paradigm for protected areas. Tour. Manag. 2017, 60, 140-146. [CrossRef]

20. Kowalczyk, M. Wskaźniki zrównoważonego rozwoju turystyki. Człowiek I Środowisko 2011, 35, 3-4.

21. Kapera, I. Rozwój Zrównoważony Turystyki. Problemy Przyrodnicze, Społeczne I Gospodarcze na Przykładzie Polski; Oficyna Wydawnicza AFM: Kraków, Poland, 2018; ISBN 978-83-66007-28-4.

22. European Tourism Indicators System for Sustainable Destination Management | Internal Market, Industry, Entrepreneurship and SMEs. Available online: https://ec.europa.eu/growth/sectors/tourism/offer/sustainable/ indicators_en (accessed on 20 March 2020).

23. Dymond, S.J. Indicators of Sustainable Tourism in New Zealand: A Local Government Perspective. J. Sustain. Tour. 1997, 5, 279-293. [CrossRef]

24. Ceron, J.-P.; Dubois, G. Tourism and Sustainable Development Indicators: The Gap between Theoretical Demands and Practical Achievements. Curr. Issues Tour. 2003, 6, 54-75. [CrossRef]

25. Tanguay, G.A.; Rajaonson, J.; Therrien, M.-C. Sustainable tourism indicators: Selection criteria for policy implementation and scientific recognition. J. Sustain. Tour. 2013, 21, 862-879. [CrossRef] 
26. Huhtala, M. Assessment of the local economic impacts of national park tourism: The case of Pallas-Ounastunturi National Park. For. Snow Landsc. Res. 2007, 223-238.

27. Huhtala, M.; Kajala, L.; Vatanen, E. Local Economic Impacts of National Park Visitors Spending: The Development Process of an Estimation Method; Working Papers of the Finnish Forest Research Institute; Finnish Forest Research Institute: Vantaa, Finland, 2010.

28. Reihanian, A.; Hin, T.W.; Kahrom, E.; Binti Mahmood, N.Z. A framework for implementing sustainable tourism in national parks of Iran: Development and use of sustainable tourism indica-tors in Boujagh National Park, Iran. Casp. J. Environ. Sci. 2015, 13, 41-52.

29. Mihanyar, P.; Rahman, S.A.; Aminudin, N. Investigating the Effect of National Park Sustainability on National Park Behavioral Intention: Kinabalu National Park. Procedia Econ. Financ. 2016, 37, 284-291. [CrossRef]

30. Kruczek, Z.; Przybyło-Kisielewska, K. Tourist traffic in national parks and consequences of excessive frequency of visitors. In Parki Narodowe I Otoczenie Społeczno-Gospodarcze: Skazani na Dialog; Walas, B., Pasierbek, T., Sobczuk, J., Pawlusiński, R., Niznansky, B., Nemethy, S., Eds.; Wyższa Szkoła turystyki i Ekologii: Sucha Beskidzka, Poland, 2019; pp. 161-170. ISBN 978-83-947044-1-4.

31. Rogowski, M. Assessing the tourism carrying capacity of hiking trails in the Szczeliniec Wielki and Błedne Skały in Stołowe Mts. National Park. For. Res. Pap. 2019, 80, 125-135. [CrossRef]

32. Mazurkiewicz, L. Prosta metoda oceny poziomu rozwoju turystyki zrównoważonej (na przykładzie turystyki zdrowotnej). Zesz. Nauk. Tur. I Rekreac. 2018, 1, 7-18.

33. Podawca, K. Planowanie przestrzenne gmin a zagospodarowanie przestrzenne parków narodowych. Acta Sci. Pol. Archit. 2006, 5, 97-110.

34. Local Data Bank of Statistics in Poland. Available online: https://bdl.stat.gov.pl/BDL/start (accessed on 10 February 2020).

35. Hadzik, A.; Hadzik, A. The chosen aspects of sustainable tourism on country health resort areas. Infrastruct. Ecol. Rural Areas 2008, 2018/02, 287-296.

36. Para, A. Zasady zrównoważonego rozwoju turystyki-bariery i szanse dla branży turystycznej, Zrównoważony rozwój turystyki w regionach Polski. Wyższa Szkoła Tur. I Języków Obcych 2013, 11, 5-16.

37. Krupa, J. Działania proekologiczne w turystyce szansą na jej zrównoważony rozwój. Zesz. Nauk. Tur. I Rekreac. 2014, 1, 5-23.

38. Mrozik, K.; Napierała, M.; Idczak, P. Ländliche Gebiete als die Herausforderung der Siedlungswasserwirtschaft in Polen. Wasserwirtschaft 2020, 38-45. [CrossRef]

39. Śleszyński, P.; Deręgowska, A.; Kubiak, Ł.; Sudra, P.; Zielińska, B. Analiza Stanu I Uwarunkowań Prac Planistycznych W Gminach W 2017 Roku; Instytut Geografii i Przestrzennego Zagospodarowania PAN: Warszawa, Poland, 2018.

40. Hurkała, T. Szczawnica jako przykład rozwoju zrównoważonego gminy uzdrowiskowej. J. Ecol. Health 2010, 14, 169-175.

(C) 2020 by the authors. Licensee MDPI, Basel, Switzerland. This article is an open access article distributed under the terms and conditions of the Creative Commons Attribution (CC BY) license (http://creativecommons.org/licenses/by/4.0/). 